Research Paper

\title{
Plasma Exosomal miRNA-139-3p is a Novel Biomarker of Colorectal Cancer
}

\author{
Wanchao Liu ${ }^{1 *}$, Dianyu Yang2², Longmei Chen ${ }^{1}$, Qingqing Liu ${ }^{1}$, Wenhui Wang ${ }^{1}$, Zhenghua Yang ${ }^{1}$, Anquan \\ Shang ${ }^{2}$, Wenqiang Quan ${ }^{2}$ and Dong $\mathrm{Li}^{2}{ }^{\bowtie}$ \\ 1. Department of Clinical Laboratory, Baoshan District Hospital of Integrated Traditional Chinese and Western Medicine, Shanghai, 201999, China. \\ 2. Department of Clinical Laboratory, Shanghai Tongji Hospital, Tongji University School of Medicine, Shanghai, 200065, China. \\ *These authors contributed equally as first authors. \\ $\bowtie$ Corresponding authors: Wenqiang Quan, E-mail: qwq@tongji.edu.cn and Dong Li, E-mail: lidong@tongii.edu.cn; Department of Clinical Laboratory, \\ Shanghai Tongji Hospital, Tongji University School of Medicine, 200065 Shanghai, China. Tel: +86 (021) 66111425; Fax: +86 (021) 56611814.
}

(c) The author(s). This is an open access article distributed under the terms of the Creative Commons Attribution License (https://creativecommons.org/licenses/by/4.0/). See http:/ /ivyspring.com/terms for full terms and conditions.

Received: 2020.03.02; Accepted: 2020.05.29; Published: 2020.06.15

\begin{abstract}
Objectives: This study investigated plasma exosomal miRNA-139-3p as a blood-based biomarker for the early diagnosis and metastasis monitoring of colorectal cancer (CRC).

Patients and Methods: Exosome-rich fractions were isolated from the plasma of $80 \mathrm{CRC}$ patients, and 23 controls using a kit method. We then used real-time polymerase chain reaction (RT-qPCR) to detect miR-139-3p levels in all subjects to evaluate expression levels and the predictive value of plasma exosomal miR-139-3p in CRC. We also collected clinicopathological data to explore correlations between abnormal miR-139-3p expression and clinicopathological parameters.

Results: When compared with healthy controls, exosomal miR-139-3p expression levels in CRC patients were significantly down-regulated. Furthermore, these expression levels were lower in metastatic colorectal cancer (mCRC) and submucosal patients. Receiver operating characteristic (ROC) curve analysis showed that exosomal miR-139-3p levels were differentiated between CRC patients and healthy controls, as well as between non-metastatic and metastatic patients.
\end{abstract}

Conclusion: Our findings show that decreased exosomal miR-139-3p expression levels in CRC patient plasma may act as a novel biomarker for the early diagnosis and metastasis monitoring in CRC.

Key words: Exosomes, miR-139-3p, colorectal cancer, metastasis

\section{Introduction}

Globally, CRC is a major factor in cancer mortality and morbidity [1]. When initially diagnosed, approximately $21 \%$ of patients will have metastatic disease, and over 50\% will develop metastases in the course of the disease [2]. These factors make CRC one of the deadliest classes of cancer. Therefore, screening procedures are essential in reducing $\mathrm{CRC}$ morbidity and mortality. Currently, several CRC screening method exist: stool testing, carcinoembryonic antigen (CEA), colonoscopy, colonography, computed tomography (CT), and double-contrast barium enemas for detecting cancer and precancerous lesions [3]. However, some of these methods are invasive, or have limited sensitivity and specificity. To address these clinical challenges, reliable and non-invasive diagnostic biomarker discovery is paramount.

Exosomes are nano-sized vesicles (30-150 nm in diameter), released into the extracellular environment by the fusion of multivesicular bodies with plasma membrane, in the blood [4]. They are surrounded by a lipid bilayer, which carries a variety of biomolecules including, glycans, proteins, metabolites, lipids, DNA and RNA [5]. Several studies have shown that exosomes express high levels of miRNAs, and that they contribute to chemo-resistance, immunomodulation and metastasis in various tumor types [6]. Similarly, previous studies have indicated that cells 
actively secrete miRNAs into the extracellular environment via exosomes, and that these molecules function as biological messengers, communicating between cells [7-9]. It is believed that exosomal miRNAs reflect some of the physiological changes and disease progression mechanisms in the human body. Moreover, the exosomal outer membrane prevents RNA degradation during in vivo circulation. Therefore, the discovery of effective biomarkers in exosome cargoes for early detection of cancer has aroused widespread interest in the scientific community.

In this study, we selected the plasma exosomal miRNA (miRNA-139-3p), identified by RNA sequencing. We investigated and validated this marker in 80 CRC patients, and 23 healthy controls, and evaluated its diagnostic potential by comparing clinical and pathological characteristics in these patients. Our data suggests that plasma exosomal miRNA-139-3p can be used as a complement to existing biomarker predictors to advance the differential diagnosis of CRC.

\section{Materials \& Methods}

\section{Patients \& clinical samples}

We collected peripheral blood samples from patients with CRC who underwent surgical resection at Tongji Hospital of Tongji University, from July 2016 to April 2018. All patients were confirmed by pathologist after operation. The ethics committee at Tongii Hospital approved this study, allowing us to obtain informed consent from each patient, prior to study commencement. All patients signed consent sheets. We finally recruited 80 colorectal cancer patients, and 23 healthy controls. Each blood sample was processed within $30 \mathrm{~min}$ of collection. All samples were centrifuged for $15 \mathrm{~min}$ at $3000 \times \mathrm{g}$ at $4{ }^{\circ} \mathrm{C}$, and then pumped and stored at $-80^{\circ} \mathrm{C}$. Following the AJCC Cancer Staging Manual, (7th edition, 2009) [10], patients were classified into groups with or without metastasis. In addition, we collected relevant clinical data from patients including age, gender, depth of invasion, tumor location and lymph node metastasis.

\section{Plasma exosome isolation}

Plasma exosomes were extracted by ExoQuick-TC ${ }^{\mathrm{TM}}$ (SBI, Palo Alto, CA) kit method. After frozen plasma samples were equilibrated to room temperature, samples were centrifuged at $3000 \times \mathrm{g}$ for $15 \mathrm{~min}$ to remove residual cells and cell debris. $250 \mu \mathrm{l}$ supernatant was removed into a new tube, to which $63 \mu$ ExoQuick reagent was added. The tube was thoroughly mixed and incubated at room temperature for $30 \mathrm{~min}$. This was followed by centrifugation at $1500 \times \mathrm{g}$ for $30 \mathrm{~min}$, after which the supernatant was discarded. This step was repeated for another $5 \mathrm{~min}$, after which the supernatant was discarded again. Finally, the remaining pellet was deemed as the exosome fraction.

\section{Transmission Electron Microscopy (TEM)}

A $20 \mu \mathrm{l}$ exosome aliquot suspension was placed onto a 200-mesh carbon coated copper grid for $2 \mathrm{~min}$. After excess liquid was removed using filter paper, the grid was negatively stained with a 3\% tungsten phosphate solution at room temperature for $3 \mathrm{~min}$. The copper mesh was washed five times in double distilled water, and allowed to dry naturally at room temperature. The sample was observed and photographed under the transmission electron microscope (Thermo-Fischer, Waltham, MA, USA).

\section{Nanosight particle tracking analysis (NTA)}

To identify the size distribution of isolated particles, a NanoSight LM10 system (Malvern Instruments Ltd. Malvern, UK) was used to size the diluted exosomes. Exosome concentration and particle size was determined by the NanoSight LM10 system software.

\section{Western blot analysis}

Diluted exosomes were added to sodium dodecyl sulfate (SDS) buffer and boiled for western blot analysis. Primary antibodies for TSG101, CD63 and CD9 were obtained from Santa Cruz Biotechnology, Inc. (Texas, USA). Secondary antibodies were rabbit-anti-mouse from Dako (Carpinteria, CA, USA) or HRP-conjugated goat anti-rabbit antibodies from Santa Cruz Biotechnology, Inc. (Texas, USA).

\section{Total RNA extraction from plasma exosomes}

Total RNA from exosomes was isolated using the miRNeasy Micro kit (QIAGEN, Germany) following the manufacturers' instructions. We determined RNA quality and quantity on the Agilent Bioanalyzer 2100 System (Agilent Technologies, CA, USA), following standard procedures.

\section{Quantitative real-time PCR}

RT-qPCR was used to quantitatively assess miR-139-3p expression levels in plasma exosomes. First, apply exosome-miRNAs as template, using TagMan MicroRNA Reverse Transcription Kit (Takara, China) to reverse miRNA to cDNA. The systems are as follows: Total RNA50-500ng, dNTP mix (10mM) 0.5ul, MMLVReverse Transcriptase (200U/ul) 0.5ul, RT primers $((10 \mu \mathrm{M}) 1 \mathrm{ul}, 5 \times \mathrm{RT}$ Buffer 5ul, RNase Inhibitor (20U/ul) 0.25ul, RNase free water up to 20ul. Polymerase Chain Reaction(PCR) conditions were: $25^{\circ} \mathrm{C} 30 \mathrm{~min}, 42{ }^{\circ} \mathrm{C} 45 \mathrm{~min}, 85^{\circ} \mathrm{C}$ 
10min to reverse miRNA to cDNA. Then apply cDNA as template, performed Realtime PCR using the Permix Ex TaqTM Kit (Takara, China) on an ABI 7300 (Applied Biosystems, Singapore) for quantification. The miR-139-3p F primer: 5'-TGGAGACGCGGCCCT GT-3'; the miR-139-3p R primer: 5'-TATGCTTGTTCT CGTCTCTGTGTC-3'. The total systems were: TaqMan Master Mix II 10.0ul, F+P primers1.0ul, cDNA+RNase-free water 9.0ul. Quantitative PCR conditions were: pre-denaturation at $95{ }^{\circ} \mathrm{C}, 10 \mathrm{~min}$ followed by $95{ }^{\circ} \mathrm{C} 12 \mathrm{~s}, 62{ }^{\circ} \mathrm{C} 40 \mathrm{~s}$, totally 40 cycles. Use small nuclear RNA U6 (U6 snRNA) as internal reference, applying the formula: $\mathrm{F}=2^{-\Delta \Delta \mathrm{CT}}$ to calculate the fold-change in the expression of the above miRNAs.

\section{Statistical analysis}

Data were represented by the mean \pm standard deviation (SD), and compared using independent $\mathrm{T}$ tests, Wilcoxon tests and Kruskal-Wallis tests, as appropriate. We set the level of statistical significance at a two-sided to $P<0.05$. Diagnostic values were assessed by receiver operating characteristic (ROC) curve analysis, and by computing the area under the curve (AUC), with confidence intervals (CI). We completed all analyses using SPSS (IBM Corp., Armonk, NY, USA) and GraphPad Prism 8 (GraphPad Software, Inc., La Jolla, CA, USA).

Table 1. Clinical characteristic of the patients

\begin{tabular}{ll}
\hline & Validation Cohort \\
\hline Total number & 80 \\
Age (years) & \\
Median (min max) & $66(41 \sim 93)$ \\
Gender & \\
Male & $46(57.5 \%)$ \\
Female & $34(42.5 \%)$ \\
Tumor location & \\
Left & $52(65.0 \%)$ \\
Right & $28(35.0 \%)$ \\
Tumor size & \\
$<2$ cm & $19(23.8 \%)$ \\
$\geq 2$ cm & $61(76.2 \%)$ \\
Invasive depth & \\
Mucosa & $18(22.5 \%)$ \\
Submucosa & $62(77.5 \%)$ \\
Nerve infiltrate & \\
+ & $18(22.5 \%)$ \\
- & $62(77.5 \%)$ \\
TNM Staging & \\
T1+T2 & $26(32.5 \%)$ \\
N3+T4 & $54(67.5 \%)$ \\
N1 & $42(52.5 \%)$ \\
N2 & $18(22.5 \%)$ \\
M1 & $20(25.0 \%)$ \\
Yetastasis (lymph nodes and distant) & $78(97.5 \%)$ \\
No & $38(47.5 \%)$ \\
\hline mean \pm SD & $42(52.5 \%)$ \\
\hline & $13.9 \pm 24.0$ \\
\hline
\end{tabular}

CEA, carcinoembryonic antigen.

\section{Results}

\section{Characterization of exosomes isolated from plasma}

To validate kit efficiency in isolating exosomes, nanoparticles were tested using NTA and TEM, and three antibody markers for extracellular vesicles. TEM images revealed oval or bowl shaped microvesicles. NTA data showed that patient plasma exosome peak sizes were between $40 \mathrm{~nm}$ and $150 \mathrm{~nm}$. Western blot analysis showed that exosomes were positive for the exosomal markers, Tsg101, CD63 and CD9. Therefore, exosome integrity and purification were confirmed.

\section{Plasma exosome-derived miR-139-3p levels at different CRC stages}

The clinical stages of all 80 patients are shown in Table 1. We examined plasma exsomal miR-139-3p levels in CRC patients and healthy controls using RT-q-PCR. As shown (Fig. 2A), exosomal miR-139-3p expression levels in patient plasma were significantly down-regulated $(P<0.001)$, when compared with healthy controls. We also investigated plasma exsomal miR-139-3p levels in CRC patients at different disease stages. From Fig. 2C, exosomal miR139-3p expression levels in patient plasma were significantly lower $(P<0.001)$ in mCRC patients than non-metastatic colorectal cancer (non-mCRC) patients. However, there were no significant differences in plasma exsomal miR-139-3p levels between $\mathrm{T} 1+\mathrm{T} 2$ and T3 + T4 $(P=0.170)$ (Fig. 2B).

\section{Correlations between plasma exosomal miR-139-3p levels and CRC clinical parameters}

We investigated potential correlations between plasma exosomal miR-139-3p levels and other clinical parameters. As shown (Fig. 3C), exosomal miR-139-3p expression levels in plasma were significantly down-regulated in the submucosal group when compared with the mucosal group $(P=0.003)$. However, no significant differences in plasma exsomal miR-139-3p levels were observed for patients with different tumor locations (Fig. 3A, Left vs. Right, $P=0.167$ ), different tumor sizes (Fig. 3B, tumor size < $2 \mathrm{~cm}$ vs. tumor size $\geq 2 \mathrm{~cm}, P=0.124)$, different CEA levels (Fig. 3D, low-CEA vs. high-CEA, $P=0.134$ ), and different nerve infiltration (Fig. $3 \mathrm{E}$, negative nerve infiltration $v$ s. positive nerve infiltration, $P=0.326$ ).

\section{Diagnostic value of each marker for colorectal cancer}

We evaluated the predictive value of miR-139-3p using ROC curve analysis. As shown (Fig. 4A), the AUC (95\% CI) for exosomal miR-139-3p and CEA, as CRC diagnostic biomarkers, were 0.726 (95\% CI; 
0.603-0.848) and 0.833 (95\% CI; 0.751-0.915), respectively. Fig. 4B showed that the AUC (95\% CI) for exosomal miR-139-3p and CEA for mCRC diagnoses was 0.766 (95\% CI; 0.662-0.869) and 0.676 (95\% CI; 0.552-0.800), respectively. However, when these two indicators were combined, the diagnostic performance was improved when compared to a single indicator (the AUC for the combined indicators for CRC diagnosis was 0.868 (95\% CI; 0.797-0.938), and for combined indicators for $\mathrm{mCRC}$ diagnosis, the AUC was 0.831 (95\% CI; 0.741-0.922). In addition, when we distinguished submucosal from mucosal patients, the AUC reached 0.733, which was superior to CEA.

\section{Discussion}

As a clinical challenge, the early diagnosis and metastasis monitoring of cancer has received much attention. In recent years, small extracellular vesicles have provided the potential to act as minimally invasive diagnostic tools for asymptomatic cancer patients. Exosomes isolated from the blood contain multiple proteins and nucleic acid moieties, of which miRNA has become topical in biomarker research, due to its therapeutic potential and stability [11-13]. miRNAs are protected from RNase activity thanks to several mechanisms: they are wrapped in membrane vesicles (microparticles, exosomes, and apoptotic bodies) and they are enclosed in lipoprotein complexes or associated with proteins [14-15]. Recent studies have demonstrated that exosomal miRNAs may act as novel biomarkers for cancer diagnosis, e.g. exosomal miRNA-21 and miRNA-1246 in CRC [16-18]. To date, this is the first study to examine the potential of plasma exosomal miRNA-139-3p in CRC diagnosis.

A

Control

CRC
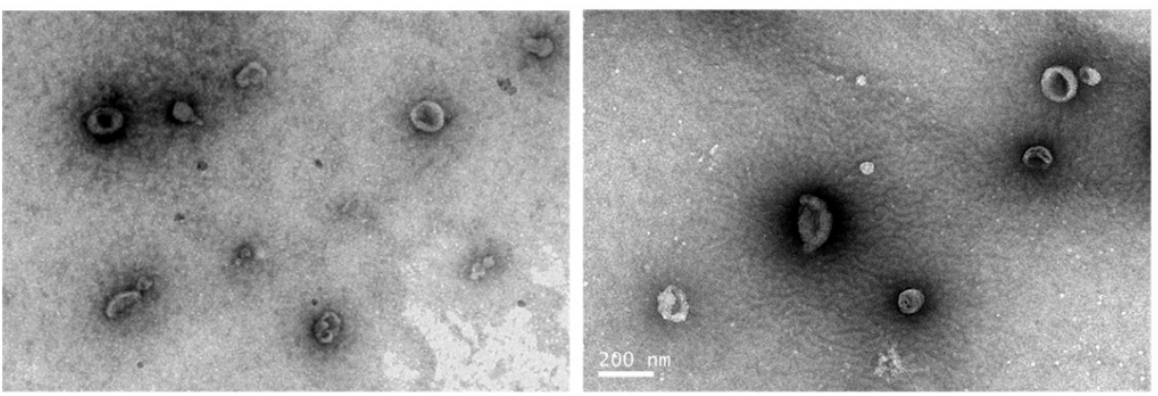

B
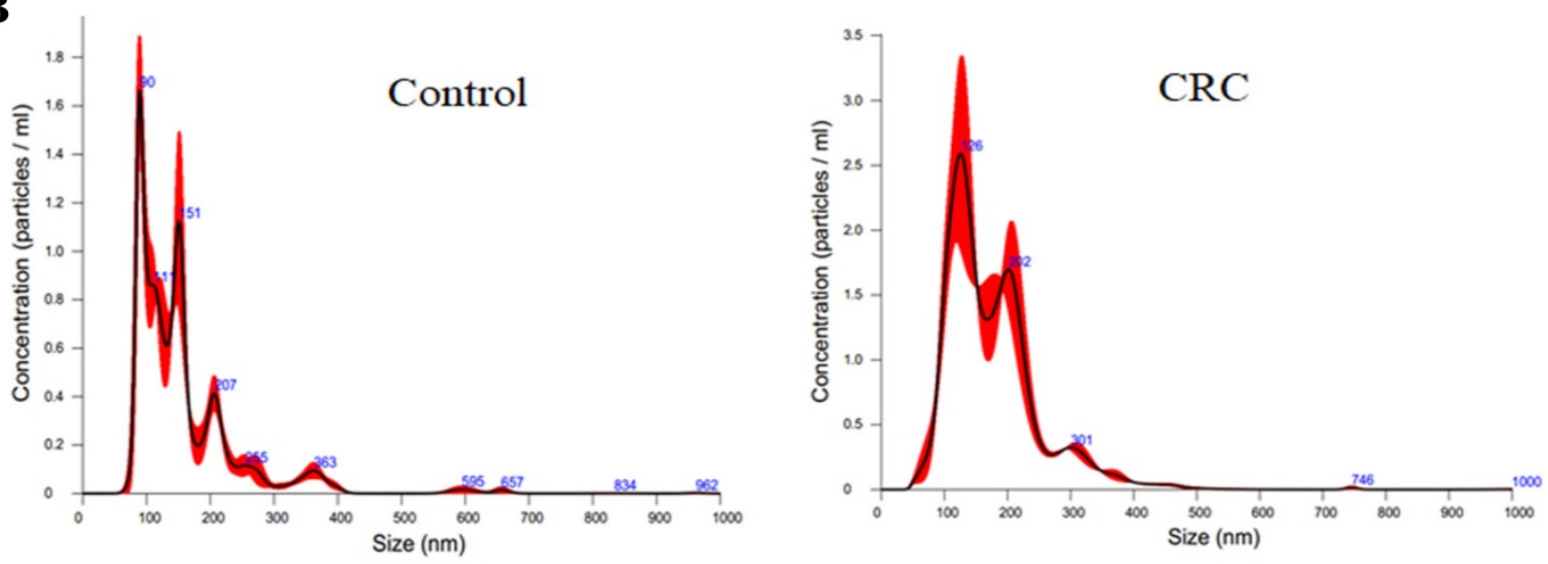

C

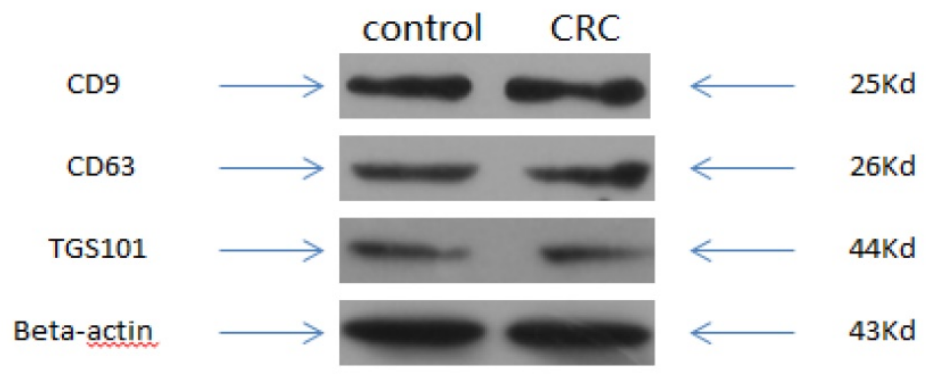

Figure 1. Characterization of exosomes isolated from plasma. (A) Transmission electron microscopy (TEM) showed the external features of the exosomes isolated from plasma. (B) Nanoparticle tracking analysis demonstrated the size distribution of the exosomes isolated from plasma. (C) Western blotting analysis of characteristic markers of extracellular vesicles, including TGS101, CD63 and CD9. 
A

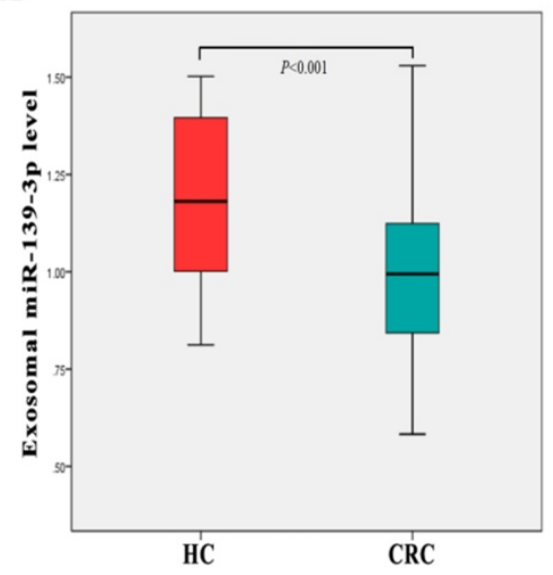

B

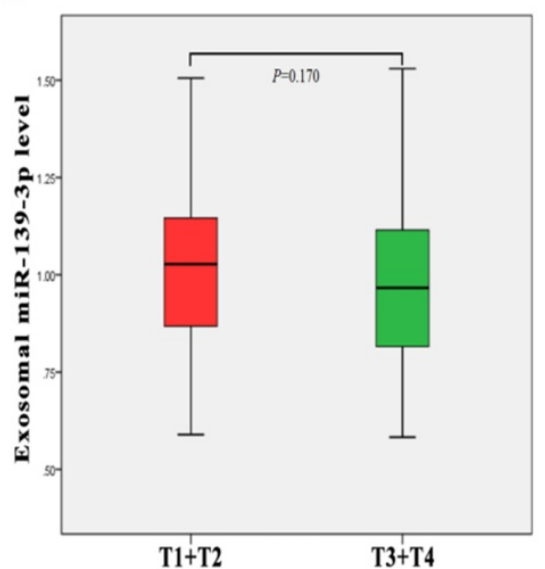

C

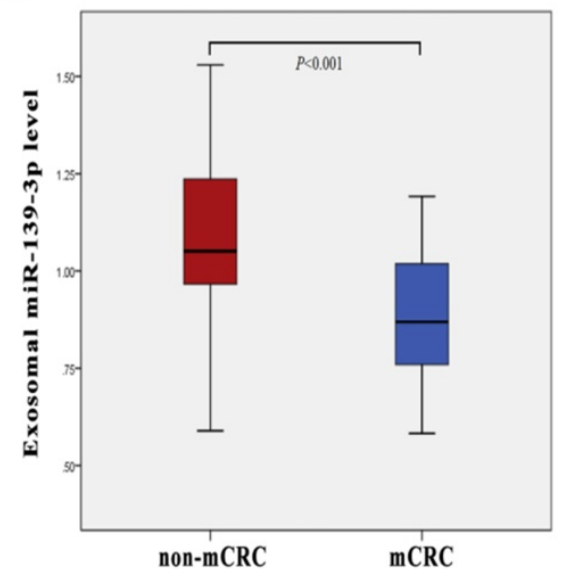

Figure 2. Plasma exosome-derived miR-139-3p levels at different colorectal cancer stages. (A) Exosomal miR-139-3p expression levels in plasma were significantly down-regulated in CRC patients when compared with healthy controls (B) No statistical differences were observed in plasma exosomal miR-139-3p levels between stages T1 + $\mathrm{T} 2$ and T3 + T4 patients. (C) Exosomal miR-139-3p expression levels in plasma were significantly down-regulated in metastatic colorectal cancer (mCRC) patients when compared with non-metastatic colorectal cancer (non-mCRC) patients.

We determined that plasma exosomal miR139-3p levels were down-regulated in CRC patients, when compared to healthy controls. Importantly, this observation distinguished CRC patients from the healthy control group. Consistent with a previous study, Liu et al., [19] reported that miR-139-3p levels in colon cancer tissues were significantly lower than those in adjacent non-cancerous tissues, and that these lower levels were significantly correlated to poor overall patient survival. Also, we observed that exosomal miR-139-3p levels in the metastatic group were significantly down-regulated when compared with the non-metastatic group. Interestingly, when compared with the infiltrating mucosa group, exosomal miR-139-3p levels in the infiltrating submucosa were significantly down-regulated, processing AUC value of 0.733. Hence, these data suggest that plasma miR-139-3p levels may be negatively correlated with CRC disease progression, which may reflect disease severity. Notably, miR139-3p was also down-regulated in other cancers including hepatocellular carcinoma and ovarian cancer [20-21]. This accumulating evidence suggests that miR-139-3p may be a potential tumor suppressor. Finally, we evaluated the diagnostic efficacy of miR139-3p and CEA combinations. The AUC for the combined CRC and mCRC diagnosis was 1 and 2, respectively, suggesting exosomal miR-139-3p may be a novel auxiliary diagnostic marker for CRC diagnosis.

Recent studies observed that exosomes, acting as messengers between tumors and stromal cells, transfer miRNAs from donor to adjacent cells [22]. This interaction may induce reprogrammed gene expression in target cells and alter tumor growth, metastasis, and epithelial-mesenchymal transformation [23]. For instance, Qi et al., [24] demonstrated that exosomal miR-660-5p promotes tumor proliferation and viability by targeting KLF9, which leads to NSCLC progression. Moreover, Wang et al., [25] reported that hypoxic tumor-derived exosomal miR-301a mediates M2 macrophage polarization via the PTEN/PI3K $\gamma$ signaling pathway to promote pancreatic cancer metastasis. However, the exosomal miR-139-3p mediated mechanism in $\mathrm{CRC}$ is still unclear.

To date, several studies have reported on the role of tissue or cell-derived miR-139-3p in other cancers, including hepatocellular carcinoma, ovarian cancer and glioma. Zou et al., [20] showed that miR-139-3p inhibited the metastatic process in HCC, through the down-regulation of ANXA2R expression. Xue et al., [21] observed that miR-139-3p inhibited ovarian cancer cell progression by inhibiting ELAVL1 expression. Furthermore, Shi et al., [26] found that miR-139-3p was implicated in glioblastoma growth and metastasis inhibition by targeting NOB1, while another study found that miR-139-3p inhibited human glioma cell invasion, proliferation, and migration via the targeting of MDA-9/syntenin [27]. These data suggest that miR-139-3p cancer regulatory mechanisms may involve multiple pathways, which require further investigation.

There were several limitations to this study. Firstly, there were no long-term clinical follow-up data for each CRC patient, thereby limiting the long term prognostic value of exosomal miR-139-3p. Secondly, due to the limited number of patients, the results must be confirmed with larger prospective studies in future. Thirdly, we did not explore the mechanism of exosomal miR-139-3p expression in $\mathrm{CRC}$, however this is anticipated in future work. 
A

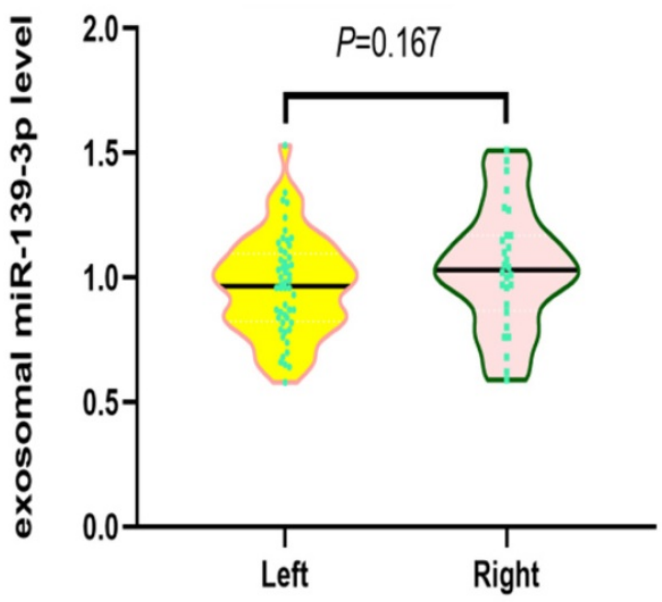

C

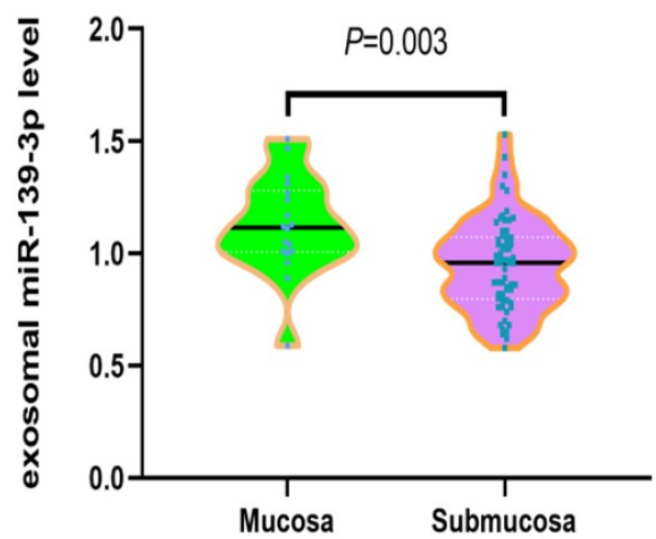

B

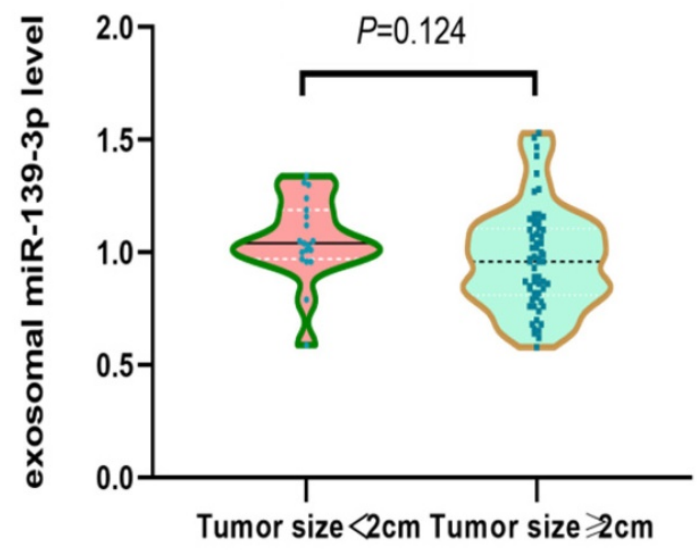

D

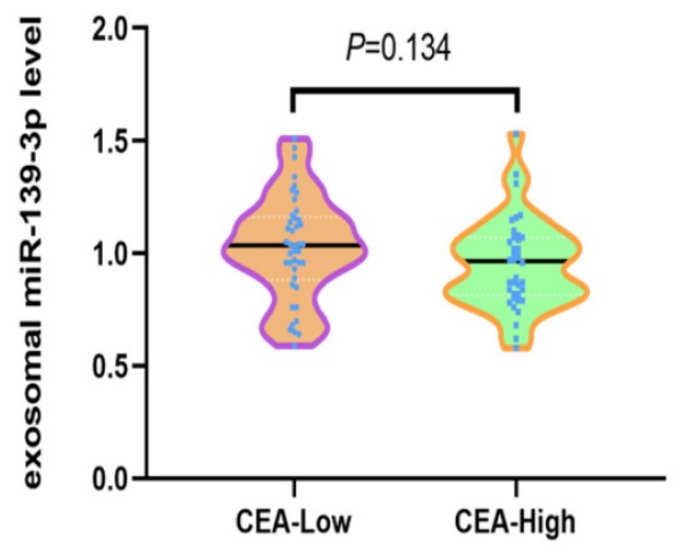

$\mathbf{E}$

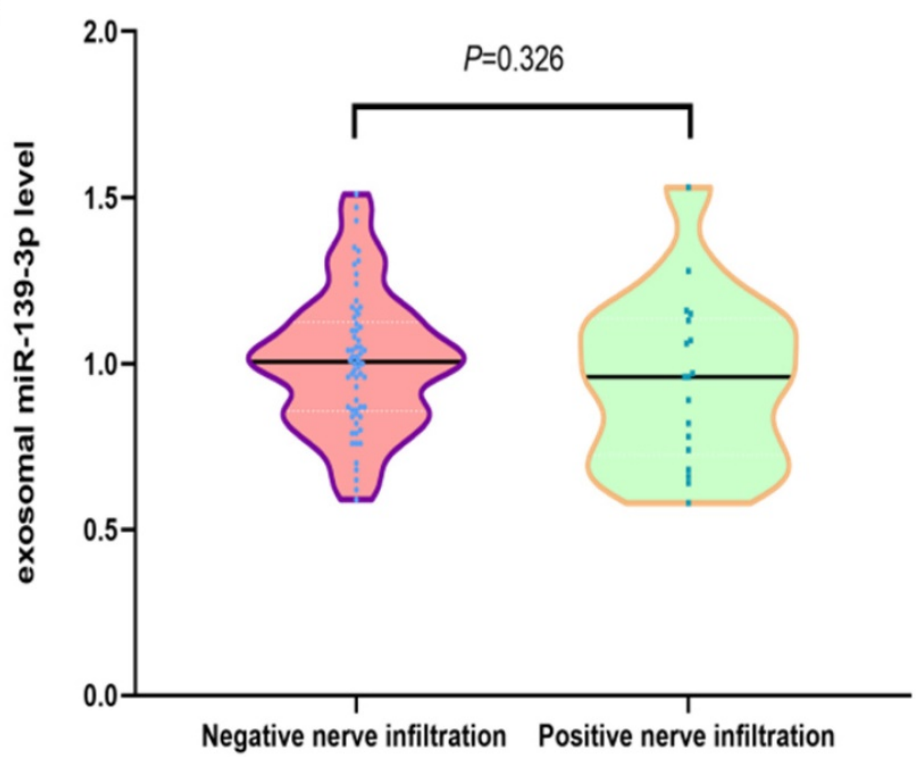

Figure 3. Correlations between plasma exosomal miR-139-3p levels and CRC clinical parameters. (A) No statistically significant differences were observed between left and right tumor locations $(P=0.167)$. (B) No statistically significant differences were found between tumor size; $<2 \mathrm{~cm}$ and $\geq 2 \mathrm{~cm}(P=0.124)$. $(C)$ Exosomal miR-139-3p expression levels in plasma were significantly down-regulated in the submucosal group when compared with the mucosal group $(P=0.003)$. (D) No statistical differences were observed in plasma exosomal miR-139-3p levels between low CEA and high CEA levels $(P=0.134)$. (E) No statistical differences were observed in plasma exosomal miR-139-3p levels between negative and positive nerve infiltration $(P=0.326)$. 

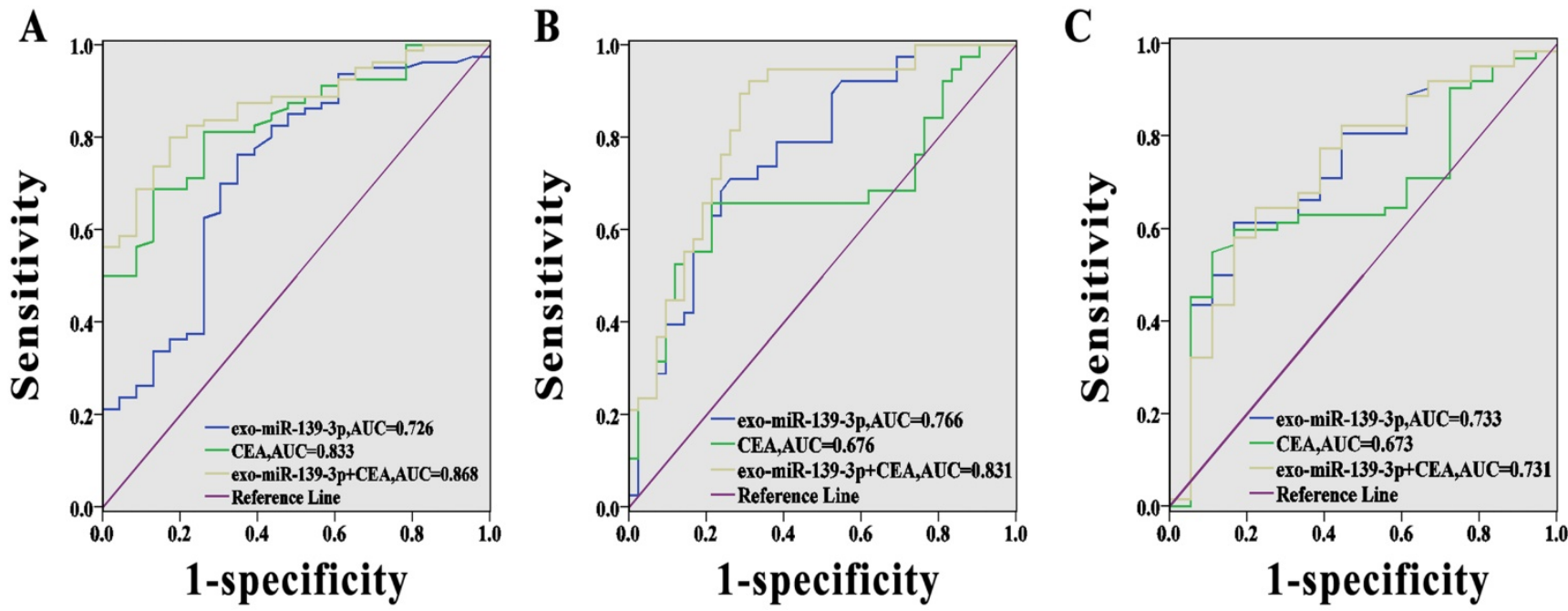

Figure 4. Diagnostic power of each marker for CRC. (A) The AUC for exosomal miR-139-3p, CEA, and combined indicators for CRC diagnosis were $0.726,0.833$ and 0.868 , respectively. (B) The AUC for exosomal miR-139-3p, CEA and combined indicators for mCRC diagnosis were $0.766,0.676$ and 0.831 , respectively. (C) The predictive value of exosomal miR-139-3p was better than CEA in terms of differentiating submucosal from mucosal patients.

\section{Conclusions}

Plasma exosomal miR-139-3p was significantly down-regulated in CRC patients, and was associated with disease progression. Our study clearly indicated that exosomal miR-139-3p may be a promising blood-based biomarker for the early diagnosis and metastasis monitoring of CRC. As a non-invasive biomarker, this molecule has great potential as an auxiliary biomarker in the diagnosis of CRC.

\section{Acknowledgements}

This work was supported by the Baoshan District Science and Technology Committee of Shanghai (18-E-14), the National Natural Science Foundation of China (81873975, 81802084, 81974314, 81902984), the Excellent Academic Leader Training Program of Shanghai Health System (2018BR31), the Medical Guidace Science and Technology Support Project of Shanghai (19411964800), the Natural Science Foundation of Shanghai (19ZR1448800).

\section{Competing Interests}

The authors have declared that no competing interest exists.

\section{References}

1. Torre LA, Bray F, Siegel RL, Ferlay J, Lortet-Tieulent J, Jemal A. Global cancer statistics. 2012. CA Cancer J Clin. 2015; 65: 87-108.

2. Stangl R, Altendorf-Hofmann A, Charnley RM, Scheele J. Factors influencing the natural history of colorectal liver metastases. Lancet. 1994:343: 1405-10.

3. Rex DK, Boland CR, Dominitz JA, et al. Colorectal Cancer Screening: Recommendations for Physicians and Patients From the U.S. Multi-Society Task Force on Colorectal Cancer. Gastroenterology. 2017:153: 307-323.

4. Van Niel G, Porto-Carreiro I, Simoes S, Raposo G. Exosomes: a common pathway for a specialized function. J Biochem. 2006;140: 13-21.

5. Mathieu M, Martin-Jaular L, Lavieu G, Théry C. Specificities of secretion and uptake of exosomes and other extracellular vesicles for cell-to-cell communication. Nat Cell Biol. 2019;21: 9-17.
6. Fang T, Lv H, Lv G, et al. Tumor-derived exosomal miR-1247-3p induces cancer-associated fibroblast activation to foster lung metastasis of liver cancer. Nat Commun. 2018; 9: 191

7. Resnick KE, Alder H, Hagan JP, Richardson DL, Croce CM, Cohn DE. The detection of differentially expressed microRNAs from the serum of ovarian cancer patients using a novel real-time PCR platform. Gynecol Oncol. 2009;112: 55-9

8. Wang LG, Gu J. Serum microRNA-29a is a promising novel marker for early detection of colorectal liver metastasis. Cancer Epidemiol. 2012;36: e61-7.

9. Brase JC, Wuttig D, Kuner R, Sültmann H. Serum microRNAs as non-invasive biomarkers for cancer. Mol Cancer. 2010;9: 306.

10. Edge SB, Byrd DR, Compton CC, et al. AJCC Cancer Staging Manual. 7th ed. NEW York:Springer,2010.

11. Srivastava A, Filant J, Moxley KM, Sood A, McMeekin S, Ramesh R. Exosomes: a role for naturally occurring nanovesicles in cancer growth, diagnosis and treatment. Curr Gene Ther. 2015;15: 182-92.

12. Ge Q, Zhou Y, Lu J, Bai Y, Xie X, Lu Z. miRNA in plasma exosome is stable under different storage conditions. Molecules. 2014;19: 1568-75.

13. Koga $Y$, Yasunaga M, Moriya $Y$, et al. Exosome can prevent RNase from degrading microRNA in feces. J Gastrointest Oncol. 2011;2: 215-22.

14. Callis TE, Pandya K, Seok HY, et al. MicroRNA-208a is a regulator of cardiac hypertrophy and conduction in mice. J Clin Invest. 2009;119: 2772-86.

15. Zernecke A, Bidzhekov K, Noels H, et al. Delivery of microRNA-126 by apoptotic bodies induces CXCL12-dependent vascular protection. Sci Signal. 2009;2: ra81.

16. Liu Q, Yu Z, Yuan S, et al. Circulating exosomal microRNAs as prognostic biomarkers for non-small-cell lung cancer. Oncotarget. 2017;8: 13048-13058.

17. Zhu M, Huang $Z$, Zhu D, et al. A panel of microRNA signature in serum for colorectal cancer diagnosis. Oncotarget. 2017; 8: 17081-17091.

18. Ogata-Kawata $H$, Izumiya $M$, Kurioka $D$, et al. Circulating exosomal microRNAs as biomarkers of colon cancer. PLoS One. 2014;9: e92921.

19. Liu X, Duan B, Dong $Y$, et al. MicroRNA-139-3p indicates a poor prognosis of colon cancer. Int J Clin Exp Pathol. 2014; 7: 8046-52.

20. Zou ZC, Dai M, Huang ZY, et al. MicroRNA-139-3p suppresses tumor growth and metastasis in hepatocellular carcinoma by repressing ANXA2R. Oncol Res. 2018 . doi: 10.3727/096504018X15178798885361

21. Xue F, Li QR, Xu YH, Zhou HB. MicroRNA-139-3p Inhibits The Growth And Metastasis Of Ovarian Cancer By Inhibiting ELAVL1. Onco Targets Ther. 2019;12: 8935-8945.

22. Donnarumma E, Fiore D, Nappa M, et al. Cancer-associated fibroblasts release exosomal microRNAs that dictate an aggressive phenotype in breast cancer. Oncotarget. 2017;8: 19592-19608.

23. He S, Li Z, Yu Y, et al. Exosomal miR-499a-5p promotes cell proliferation, migration and EMT via mTOR signaling pathway in lung adenocarcinoma. Exp Cell Res. 2019;379(2): 203-213.

24. Oi $Y$, Zha W, Zhang W. Exosomal miR-660-5p promotes tumor growth and metastasis in non-small cell lung cancer. J BUON. 2019; 24: 599-607.

25. Wang X, Luo G, Zhang K, et al. Hypoxic Tumor-Derived Exosomal miR-301a Mediates M2 Macrophage Polarization via PTEN/PI3KY to Promote Pancreatic Cancer Metastasis. Cancer Res. 2018;78: 4586-4598.

26. Shi L, Yuan Y, Li HY. MicroRNA-139-3p suppresses growth and metastasis of glioblastoma via inhibition of NIN1/RPNI2 binding protein 1 homolog. Eur Rev Med Pharmacol Sci. 2019;23: 4264-4274. 
27. Tian $\mathrm{W}, \mathrm{Wu}$ W, Li X, Rui X, Wu Y. MiRNA-139-3p inhibits the proliferation, invasion, and migration of human glioma cells by targeting MDA-9/syntenin. Biochem Biophys Res Commun. 2019;508: 295-301. 